\title{
Resonance Fluorescence Spectrum Near Photonic Bandgap
}

\author{
Ray-Kuang Lee and Yinchieh Lai \\ Institute of Electro-Optical Engineering, National Chiao-Tung University, Hsinchu, Taiwan
}

\begin{abstract}
We theoretically calculate the resonance fluorescence spectrum from a two-level atom which is embedded in a photonic bandgap crystal and is resonantly driven by a classical pump light. Non-Markovian noises caused by the non-uniform distribution of photon density states near the photonic bandgap are taken into account by a new approach which linearizes the optical Bloch equations by using the Liouville operator expansion. These linearized equations can be solved directly in the Fourier domain to obtain the correlation functions of the atomic operators and then the fluorescence spectrum from the atom. We find that if the atomic energy level is far from the bandgap, fluorescence spectra with Mollow triplets are observed. When the atomic energy level is near the bandgap, the relative magnitude and the number of the fluorescence peaks are found to be varied according to the wavelength offset.
\end{abstract}

Keywords: Fluorescence-atoms, Photonic bandgap materials, Cavity quantum electrodynamics

\section{INTRODUCTION}

It has been well known that the spontaneous emission from an excited atom can be modified by the electromagnetic reservoir that surrounds the atom. ${ }^{1}$ Within a periodic dielectric structure (or especially the photonic bandgap crystal), ${ }^{2,3}$ the properties of the photon field reservoir as well as the properties of the spontaneous emission can be modified dramatically. In photonic bandgap crystals, the propagation of light is prohibited within a certain range of wavelengths (the bandgap) due to the lack of available photon states. When above or below the bandgap, the density of photon states starts to increase from zero and has a non-unifoum distribution. This is in contrast to the free space situation where the distribution of the photon density states is more uniform. It is the aim of this paper to investigate the impacts of such non-unifoum photon state distribution upon the characteristics of the resonance fluorescence from an atom with its emission wavelength near the bandgap.

In contrast to the situation at free space where the Markovian approximation and the quantum regression theorem are valid, the atom-photon interaction in photonic bandgap materials is in general non-Markovian and has been found to exhibit many interesting new phenomena including the appearance of photon-atom bound states, ${ }^{4}$ spectral splitting, ${ }^{5}$ dark line with quantum interference effect, ${ }^{6}$ phase control of spontaneous emission, ${ }^{7}$ transparency near band edge, ${ }^{8}$ and single-atom switching. ${ }^{9}$ In this paper we will investigate the properties of the resonance fluorescence emitted by a two-level atom embedded in a photonic bandgap crystal and driven by a classical pumping light. Due to the non-Markovian nature of the atom-field interaction in this case, the Born-Markovian approximation which is usually used in quantum optics for treating atom-photon interaction problems can not be directly applied here. To overcome this difficulty, we first derive the generalized optical Bloch equations without any approximation and then use the Liouville operator expansion to approximate the nonlinear terms while still keep the non-Markovian memory functions. By solving the simplified linear equations in the Fourier domain directly we can calculate the stationary two-time correlation functions of the atomic operators as well as the spectral distribution of the resonance fluorescence without too much difficulty. When the emission frequency of the atom is far from the band edge, the results are found to agree with the results of Mollow $^{10}$ for the case at free space. The triplet spectral shape is observed just as one will expect intuitively. But when we change the emission wavelength of the atom to be close to the band edge, both the number of the peaks as well as their profiles are found to be varied depending on the wavelength offset between the atomic transition wavelength and the band edge. The details of these results as well as the theoretical formulation for calculating them will be presented in the following sections respectively.

E-mail: yclai@mail.nctu.edu.tw 


\section{GENERALIZED OPTICAL BLOCH EQUATIONS}

The system we consider consists of a single two-level atom embedded in a photonic bandgap crystal and driven by a classical pump light. The transition frequency of the atom and the frequency of the pumping light are denoted to be $\omega_{a}$ and $\omega_{L}$ respectively. In this model we treat the photon states in the photonic bandgap crystal as the background reservoir and use the Jaynes-Cummings model to describe the atom-photon interaction. The Hamiltonian for our system can be written as:

$$
H=\frac{\hbar}{2} \omega_{a} \sigma_{z}+\hbar \sum_{k} \omega_{k} a_{k}^{\dagger} a_{k}+\frac{\Omega}{2} \hbar\left(\sigma_{-} e^{i \omega_{L} t}+\sigma_{+} e^{-i \omega_{L} t}\right)+\hbar \sum_{k}\left(g_{k} \sigma_{+} a_{k}+g_{k}^{*} a_{k}^{\dagger} \sigma_{-}\right)
$$

where $a_{k}^{\dagger}$ and $a_{k}$ are the creation and annihilation operators of the background photon reservoir, $\Omega$ is the Rabiflopping frequency of the atom under the external pumping light and also represents the relative magnitude of the pumping light, $\sigma_{z} \equiv(|2\rangle\langle 2|-| 1\rangle\langle 1|), \sigma_{+} \equiv|2\rangle\langle 1|=\sigma_{-}^{\dagger}$ are the usual Pauli matrices for a two-level atom, and $g_{k}$ is atom-field coupling constant.

Although the form of the coupling constant $g_{k}$ depends on the gauge one choose, in non-relativistic quantum field theories either the $e p \cdot A$ or the $-\mu \cdot E$ formula is used. In this paper we chose the $e p \cdot A$ formulation and under this formulation the coupling constant $g_{k}$ can be expressed as:

$$
g_{k}=|d| \omega_{a} \sqrt{\frac{1}{2 \hbar \epsilon_{0} \omega_{k} V}} \mathbf{d} \cdot \mathbf{E}^{*}\left(r_{0}\right)
$$

where $|d|$ is the magnitude of the atomic dipole moment, $\mathbf{d}$ is the unit vector along the direction of the dipole moment, $V$ is the volume of the quantization space, and $\epsilon_{0}$ is the Coulomb constant.

By using Eq.(1) to derive the Heisenberg equations for our system in the rotating frame with the frequency $\omega_{a}$ and by eliminating the reservoir field operators, we get the generalized Bloch equations as follow:

$$
\begin{aligned}
& \dot{\sigma}_{-}(t)=i \frac{\Omega}{2} \sigma_{z}(t) e^{-i \Delta t}+\int_{-\infty}^{t} d t^{\prime} G\left(t-t^{\prime}\right) \sigma_{z}(t) \sigma_{-}\left(t^{\prime}\right)+n_{-}(t) \\
& \dot{\sigma}_{+}(t)=-i \frac{\Omega}{2} \sigma_{z}(t) e^{i \Delta t}+\int_{-\infty}^{t} d t^{\prime} G_{c}\left(t-t^{\prime}\right) \sigma_{+}\left(t^{\prime}\right) \sigma_{z}(t)+n_{+}(t) \\
& \dot{\sigma}_{z}(t)=i \Omega\left(\sigma_{-}(t) e^{i \Delta t}-\sigma_{+}(t) e^{-i \Delta t}\right)-2 \int_{-\infty}^{t} d t^{\prime}\left[G\left(t-t^{\prime}\right) \sigma_{+}(t) \sigma_{-}\left(t^{\prime}\right)+G_{c}\left(t-t^{\prime}\right) \sigma_{+}\left(t^{\prime}\right) \sigma_{-}(t)\right]+n_{z}(t)(5)
\end{aligned}
$$

where $\Delta \equiv \omega_{L}-\omega_{a}$, and $\Delta_{k} \equiv \omega_{a}-\omega_{k}$. The two functions $G(\tau)$ and $G_{c}(\tau)$ are the memory functions of the system and are defined as $G(\tau) \equiv \sum_{k}\left|g_{k}\right|^{2} e^{i \Delta_{k} \tau} \Theta(\tau)$, and $G_{c}(\tau) \equiv \sum_{k}\left|g_{k}\right|^{2} e^{-i \Delta_{k} \tau} \Theta(\tau)$. $\Theta(\tau)$ is the Heaviside step function. Moreover, the three noise operators $n_{-}(t), n_{+}(t)$, and $n_{z}(t)$ are defined as follows:

$$
\begin{aligned}
& n_{-}(t)=i \sum_{k} g_{k} e^{i \Delta_{k} t} \sigma_{z}(t) a_{k}(-\infty) \\
& n_{+}(t)=-i \sum_{k} g_{k}^{*} e^{-i \Delta_{k} t} a_{k}^{\dagger}(-\infty) \sigma_{z}(t) \\
& n_{z}(t)=-2 i \sum_{k} g_{k} e^{i \Delta_{k} t} \sigma_{+}(t) a_{k}(-\infty)+2 i \sum_{k} g_{k}^{*} e^{-i \Delta_{k} t} a_{k}^{\dagger}(-\infty) \sigma_{-}(t)
\end{aligned}
$$

Supposing that the reservoir is in thermal equilibrium, then in the beginning the mean and correlation of the reservoir field operators will be:

$$
\begin{aligned}
& \left\langle a_{k}(-\infty)\right\rangle_{R}=\left\langle a_{k}^{\dagger}(-\infty)\right\rangle_{R}=0 \\
& \left\langle a_{k}(-\infty) a_{k^{\prime}}(-\infty)\right\rangle_{R}=0 \\
& \left\langle a_{k}^{\dagger}(-\infty) a_{k^{\prime}}^{\dagger}(-\infty)\right\rangle_{R}=0 \\
& \left\langle a_{k}^{\dagger}(-\infty) a_{k^{\prime}}(-\infty)\right\rangle_{R}=\bar{n}_{k} \delta_{k k^{\prime}} \\
& \left\langle a_{k}(-\infty) a_{k^{\prime}}^{\dagger}(-\infty)\right\rangle_{R}=\left(\bar{n}_{k}+1\right) \delta_{k k^{\prime}}
\end{aligned}
$$


where $\bar{n}_{k}$ obeys the Bose-Einstein distribution. Using the statistical characteristics of the reservoir field operators, it can be easily shown that the three noise operators $n_{-}(t), n_{+}(t)$, and $n_{z}(t)$ are zero mean with their correlation functions given below:

$$
\begin{aligned}
& \left\langle n_{-}(t)\right\rangle_{R}=\left\langle n_{+}(t)\right\rangle_{R}=\left\langle n_{z}(t)\right\rangle_{R}=0 \\
& \left\langle n_{-}(t) n_{-}\left(t^{\prime}\right)\right\rangle_{R}=\left\langle n_{+}(t) n_{+}\left(t^{\prime}\right)\right\rangle_{R}=0 \\
& \left\langle n_{-}(t) n_{+}\left(t^{\prime}\right)\right\rangle_{R}=\sum_{k}\left|g_{k}\right|^{2}\left(\bar{n}_{k}+1\right) e^{i \Delta_{k}\left(t-t^{\prime}\right)}\left\langle\sigma_{z}(t) \sigma_{z}\left(t^{\prime}\right)\right\rangle \\
& \left\langle n_{+}(t) n_{-}\left(t^{\prime}\right)\right\rangle_{R}=\sum_{k}\left|g_{k}\right|^{2} \bar{n}_{k} e^{-i \Delta_{k}\left(t-t^{\prime}\right)}\left\langle\sigma_{z}(t) \sigma_{z}\left(t^{\prime}\right)\right\rangle \\
& \left\langle n_{z}(t) n_{z}\left(t^{\prime}\right)\right\rangle_{R}=4 \sum_{k}\left|g_{k}\right|^{2}\left[\left(\bar{n}_{k}+1\right) e^{i \Delta_{k}\left(t-t^{\prime}\right)}\left\langle\sigma_{+}(t) \sigma_{-}\left(t^{\prime}\right)\right\rangle+\bar{n}_{k} e^{-i \Delta_{k}\left(t-t^{\prime}\right)}\left\langle\sigma_{-}(t) \sigma_{+}\left(t^{\prime}\right)\right\rangle\right]
\end{aligned}
$$

Eqs.(3-5) are the generalized optical Bloch equations, which are general equations and will serve as the starting point for our further derivation.

\section{MODELING OF PHOTONIC BANDGAP CRYSTALS}

To model the the photon properties of photonic bandgap crystals, one will need to know the spectral distribution of the photon states. In this paper we will use the anisotropic mode ${ }^{11}$ to describe the spectral density of photon states near the photonic band edge. For three dimensional photonic bandgap crystals, if the wavevector that corresponds to the band edge is $\mathbf{k}_{0}^{i}$, then the dispersion relation in the anisotropic model is described by the following form: $\omega_{k}=\omega_{c}+A\left|\mathbf{k}-\mathbf{k}_{0}^{i}\right|^{2}$, where $A$ is a model dependent constant and $\omega_{c}$ is the band edge frequency. Based on this dispersion relation, the corresponding density of states (DOS) is given by: $D(\omega)=\frac{1}{A^{3 / 2}} \sqrt{\omega-\omega_{c}} \Theta\left(\omega-\omega_{c}\right)$. The memory functions under this anisotropic model also can be derived:

$$
\begin{aligned}
\tilde{G}(\omega) & =\beta^{3 / 2} \frac{-i}{\sqrt{\omega_{c}}+\sqrt{\omega_{c}-\omega_{a}-\omega}} \\
\tilde{G}_{c}(\omega) & =\beta^{3 / 2} \frac{i}{\sqrt{\omega_{c}}+\sqrt{\omega_{c}-\omega_{a}+\omega}}
\end{aligned}
$$

where $\beta^{3 / 2}=\frac{\omega_{a}^{2} d^{2}}{6 \hbar \epsilon_{0} \pi A^{3 / 2}} \eta$, and we have used the space average coupling strength $\eta \equiv \frac{3}{8 \pi} \int d \Omega|\mathbf{d} \cdot \mathbf{E}|^{2}$ in the derivation.

It can be easily checked that the Full-Width-Half-Maximum (FWHM) bandwidth of the memory functions in Eq.(19) and Eq.(20) are $4 \omega_{c}$. In the optical domain, $\omega_{c} \approx 10^{14-15} \mathrm{~Hz}$, and the typical lifetime of the atom is from $10^{-3}$ sec to $10^{-9} \mathrm{sec}$, which is much longer than the response time of the memory functions. Therefore it should be possible to approximate the two-time operator products in Eqs.(3-5) by the equal time operator products with the introduction of the Liouville operator expansion to be given below. For a two-level atom system described by the Hamiltonian $H$, the time evolution of the atomic operators can be written in general as:

$$
\sigma_{i j}(t)=e^{-i \mathcal{L}\left(t-t^{\prime}\right)} \sigma_{i j}\left(t^{\prime}\right)=\sum_{n=0}^{\infty} \frac{\left(-i\left(t-t^{\prime}\right)\right)^{n}}{n !} \mathcal{L}^{n} \sigma_{i j}\left(t^{\prime}\right)
$$

where the Liouville operator $\mathcal{L}$ is defined as

$$
\left.\mathcal{L}^{n} \sigma_{i j}\left(t^{\prime}\right)=\frac{1}{\hbar^{n}}\left[\cdots\left[\sigma_{i j}\left(t^{\prime}\right), H\right], H\right], \cdots, H\right]
$$

Since in this paper we will only consider the case in which the atom is with a longer lifetime and is under weak pumping (small Rabi frequency), the time scale of the atomic evolution will be always longer than the time scale of the memory functions. Therefore under such assumptions it should be legitimate to simply apply the zero-th 
order perturbation terms. We have also checked the results obtained by using the first order perturbation terms and they only show difference when the pumping is extremely high. ${ }^{12}$ Under the zero-th order approximation, $\sigma_{z}(t) \approx \sigma_{z}\left(t^{\prime}\right)$, and $\sigma_{ \pm}(t) \approx \sigma_{ \pm}\left(t^{\prime}\right)$ and the generalized optical Bloch equations Eqs.(3-5) can be reduced to:

$$
\begin{aligned}
& \dot{\sigma}_{-}(t)=i \frac{\Omega}{2} \sigma_{z}(t) e^{-i \Delta t}-\int_{-\infty}^{t} d t^{\prime} G\left(t-t^{\prime}\right) \sigma_{-}\left(t^{\prime}\right)+n_{-}(t) \\
& \dot{\sigma}_{+}(t)=-i \frac{\Omega}{2} \sigma_{z}(t) e^{i \Delta t}-\int_{-\infty}^{t} d t^{\prime} G_{c}\left(t-t^{\prime}\right) \sigma_{+}\left(t^{\prime}\right)+n_{+}(t) \\
& \dot{\sigma}_{z}(t)=i \Omega\left(\sigma_{-}(t) e^{i \Delta t}-\sigma_{+}(t) e^{-i \Delta t}\right)-\int_{-\infty}^{t} d t^{\prime}\left[G\left(t-t^{\prime}\right)+G_{c}\left(t-t^{\prime}\right)\right]\left(1+\sigma_{z}\left(t^{\prime}\right)\right)+n_{z}(t)
\end{aligned}
$$

It is important to note that the equations are now in a linear form while the non-Markovian memory functions are still kept. Since it is now a linear problem, by using Fourier transform the modified optical Bloch equations become:

$$
\overline{\overline{\mathcal{M}}}(\omega) \cdot \overrightarrow{\mathcal{X}}(\omega)=\overrightarrow{\mathcal{X}}_{0}(\omega)
$$

where

$$
\begin{aligned}
& \overline{\overline{\mathcal{M}}}(\omega)=\left(\begin{array}{ccc}
-i(\omega+\Delta)+\tilde{G}(\omega) & 0 & -i \frac{\Omega}{2} \\
0 & -i(\omega-\Delta)+\tilde{G}_{c}(\omega) & i \frac{\Omega}{2} \\
-i \Omega & i \Omega & -i \omega+\tilde{G}(\omega)+\tilde{G}_{c}(\omega)
\end{array}\right) \\
& \overrightarrow{\mathcal{X}}(\omega)=\left(\begin{array}{c}
\tilde{\sigma}_{-}(\omega+\Delta) \\
\tilde{\sigma}_{+}(\omega-\Delta) \\
\tilde{\sigma}_{z}(\omega)
\end{array}\right) \\
& \overrightarrow{\mathcal{X}}_{0}(\omega)=\left(\begin{array}{c}
\tilde{n}_{-}(\omega+\Delta) \\
\tilde{n}_{+}(\omega-\Delta) \\
-2 \pi\left[\tilde{G}(\omega)+\tilde{G}_{c}(\omega)\right] \delta(\omega)+\tilde{n}_{z}(\omega)
\end{array}\right)
\end{aligned}
$$

where $\tilde{n}_{-}(\omega), \tilde{n}_{+}(\omega), \tilde{n}_{z}(\omega), \tilde{G}(\omega)$, and $\tilde{G}_{c}(\omega)$ are Fourier transforms of $n_{-}(t), n_{+}(t), n_{z}(t), G(t)$, and $G_{c}(t)$, respectively. The solutions of Eq.(26) are

$$
\begin{aligned}
& \tilde{\sigma}_{-}(\omega+\Delta)=\frac{\left(2 g h+\Omega^{2}\right) \tilde{n}_{-}(\omega)+\Omega^{2} \tilde{n}_{+}(\omega)+i \Omega g \tilde{n}_{z}(\omega)-i 2 \pi \Omega g\left[\tilde{G}(\omega)+\tilde{G}_{c}(\omega)\right] \delta(\omega)}{\Omega^{2}(f+g)+2 f g h} \\
& \tilde{\sigma}_{+}(\omega-\Delta)=\frac{\Omega^{2} \tilde{n}_{-}(\omega)+\left(2 f h+\Omega^{2}\right) \tilde{n}_{+}(\omega)-i \Omega f \tilde{n}_{z}(\omega)+i 2 \pi \Omega f\left[\tilde{G}(\omega)+\tilde{G}_{c}(\omega)\right] \delta(\omega)}{\Omega^{2}(f+g)+2 f g h} \\
& \tilde{\sigma}_{z}(\omega)=\frac{2 i \Omega g \tilde{n}_{-}(\omega)-2 i \Omega f \tilde{n}_{+}(\omega)+2 f g \tilde{n}_{z}(\omega)-4 \pi f g\left[\tilde{G}(\omega)+\tilde{G}_{c}(\omega)\right] \delta(\omega)}{\Omega^{2}(f+g)+2 f g h}
\end{aligned}
$$

where

$$
\begin{aligned}
& f(\omega)=-i \omega-i \Delta+\tilde{G}(\omega) \\
& g(\omega)=-i \omega+i \Delta+\tilde{G}_{c}(\omega) \\
& h(\omega)=-i \omega+\tilde{G}(\omega)+\tilde{G}_{c}(\omega)
\end{aligned}
$$

Because the two-time correlation function of the atomic dipole is proportional to the first order correlation function $g^{(1)}(\tau)$, we can obtain the fluorescence spectrum by taking the Fourier transform of the first order correlation function:

$$
\begin{aligned}
S(\omega) & =\int_{-\infty}^{\infty} d \tau g^{(1)}(\tau) e^{i \omega \tau} \\
& \propto\left\langle\tilde{\sigma}_{+}(\omega) \tilde{\sigma}_{-}(-\omega)\right\rangle_{R}
\end{aligned}
$$


By using this equation the fluorescence spectrum can be determined from Eqs.(30-31)after applying the noise correlation functions. It should be noted that here we cannot directly apply the quantum regression theorem since it is invalid for non-Markovian process.

As a check, we first use our formulation to calculate the free space case. At free space, one can assume the memory functions are delta functions since $\sum_{k}\left|g_{k}\right|^{2} e^{i \Delta_{k} t}=\Gamma \delta(t)$ with $\Gamma$ being the decay rate of the excited atom. The noise correlation functions at zero temperature are also delta-function correlated (i.e., white noises). Therefore, the fluorescence spectrum at steady state is given by:

$$
\begin{aligned}
& \left\langle\tilde{\sigma_{+}}(\omega) \tilde{\sigma_{-}}(-\omega)\right\rangle_{R}=\frac{\pi^{2} \Omega^{2}\left(\frac{\Gamma^{2}}{4}+\Delta^{2}\right)}{\frac{\Omega^{2}}{2}+\Delta^{2}+\frac{\Gamma^{2}}{4}} \delta(\omega+\Delta) \\
& +\frac{\pi \Gamma \Omega^{4}\left(\frac{\Omega^{2}}{2}+\Gamma^{2}+(\omega+\Delta)^{2}\right)}{2\left(\frac{\Omega^{2}}{2}+\Delta^{2}+\frac{\Gamma^{2}}{4}\right)\left[\Gamma^{2}\left(\frac{\Omega^{2}}{2}+\Delta^{2}+\frac{\Gamma^{2}}{4}-2(\omega+\Delta)^{2}\right)^{2}+(\omega+\Delta)^{2}\left(\Omega^{2}+\Delta^{2}+\frac{5}{4} \Gamma^{2}-(\omega+\Delta)^{2}\right)^{2}\right]}
\end{aligned}
$$

In the limit of strong on-resonance pumping $(\Omega \gg \Gamma, \Delta=0)$, Eq.(34) can be reduced to:

$$
\left\langle\tilde{\sigma_{+}}(\omega) \tilde{\sigma_{-}}(-\omega)\right\rangle_{R}=2 \pi\left\{2 \pi \frac{\Gamma^{2}}{4 \Omega^{2}} \delta(\omega)+\frac{\frac{3}{16} \Gamma}{(\omega+\Omega)^{2}+\frac{9}{16} \Gamma^{2}}+\frac{\frac{1}{4} \Gamma}{\omega^{2}+\frac{1}{4} \Gamma^{2}}+\frac{\frac{3}{16} \Gamma}{(\omega-\Omega)^{2}+\frac{9}{16} \Gamma^{2}}\right\}
$$

Then, the resonance fluorescence spectrum exhibits the Mollow triplets ${ }^{10}$ for white noise: three Lorentzian profiles with peaks in the ratio $1: 3: 1$, and widths of $\frac{3}{2} \Gamma, \Gamma$, and $\frac{3}{2} \Gamma$. This check provides a good support for our new formulation.

We now continue to calculate the case for a photonic bandgap material. By using the density of states for the photonic bandgap crystal, $D(\omega)=\frac{1}{A^{3 / 2}} \sqrt{\omega-\omega_{c}} \Theta\left(\omega-\omega_{c}\right)$, we get the following noise correlation functions in the frequency domain at the zero temperature:

$$
\begin{aligned}
& \left\langle\tilde{n}_{-}\left(\omega_{1}\right) \tilde{n}_{+}\left(-\omega_{2}\right)\right\rangle_{R}=4 \pi \beta^{3 / 2} \frac{\sqrt{\omega_{a}+\omega_{1}-\omega_{c}}}{\omega_{a}+\omega_{1}} \Theta\left(\omega_{1}+\omega_{a}-\omega_{c}\right) \delta\left(\omega_{1}-\omega_{2}\right) \\
& \left\langle\tilde{n}_{z}\left(\omega_{1}\right) \tilde{n}_{z}\left(-\omega_{2}\right)\right\rangle_{R}=4 \beta^{3 / 2} \frac{\sqrt{\omega_{a}+\omega_{1}-\omega_{c}}}{\omega_{a}+\omega_{1}}\left[2 \pi \delta\left(\omega_{1}-\omega_{2}\right)+\left\langle\tilde{\sigma}_{z}\left(\omega_{1}-\omega_{2}\right)\right\rangle_{R}\right] \Theta\left(\omega_{1}+\omega_{a}-\omega_{c}\right) \\
& \left\langle\tilde{n}_{z}\left(\omega_{1}\right) \tilde{n}_{-}\left(-\omega_{2}\right)\right\rangle_{R}=0 \\
& \left\langle\tilde{n}_{-}\left(\omega_{1}\right) \tilde{n}_{z}\left(-\omega_{2}\right)\right\rangle_{R}=4 \beta^{3 / 2} \frac{\sqrt{\omega_{a}+\omega_{1}-\omega_{c}}}{\omega_{a}+\omega_{1}}\left\langle\tilde{\sigma}_{-}\left(\omega_{1}-\omega_{2}\right)\right\rangle_{R} \Theta\left(\omega_{1}+\omega_{a}-\omega_{c}\right) \\
& \left\langle\tilde{n}_{z}\left(\omega_{1}\right) \tilde{n}_{+}\left(-\omega_{2}\right)\right\rangle_{R}=4 \beta^{3 / 2} \frac{\sqrt{\omega_{a}+\omega_{1}-\omega_{c}}}{\omega_{a}+\omega_{1}}\left\langle\tilde{\sigma}_{+}\left(\omega_{1}-\omega_{2}\right)\right\rangle_{R} \Theta\left(\omega_{1}+\omega_{L}+-\omega_{c}\right) \\
& \left\langle\tilde{n}_{+}\left(\omega_{1}\right) \tilde{n}_{z}\left(-\omega_{2}\right)\right\rangle_{R}=0
\end{aligned}
$$

Please note that these noise correlation functions are not only of the color-noise type but also with the characteristics of the photonic bandgap. After applying these noise correlation functions Eqs.(36-41) as well as the memory functions in Eqs.(19-20), we can get the fluorescence spectrum under resonant pumping from the two-time correlation function $\left\langle\sigma_{+}(t) \sigma_{-}\left(t^{\prime}\right)\right\rangle_{R}$ given in Eq.(33):

$$
\begin{aligned}
& \left\langle\tilde{\sigma_{+}}(\omega) \tilde{\sigma_{-}}(-\omega)\right\rangle_{R}=4 \beta^{3 / 2} \frac{\sqrt{\omega_{a}+\omega-\omega_{c}}}{\omega_{a}+\omega} . \\
& \frac{\pi \Omega^{4}+i \Omega^{3} g(-\omega)\left\langle\tilde{\sigma}_{-}(0)\right\rangle_{R}-i \Omega^{3} f(\omega)\left\langle\tilde{\sigma}_{+}(0)\right\rangle_{R}+\Omega^{2} f(\omega) g(-\omega)\left(2 \pi+\left\langle\tilde{\sigma}_{z}(0)\right\rangle_{R}\right)}{\left\{\Omega^{2}[f(\omega)+g(\omega)]+2 f(\omega) g(\omega) h(\omega)\right\}\left\{\Omega^{2}[f(-\omega)+g(-\omega)]+2 f(-\omega) g(-\omega) h(-\omega)\right\}} \Theta\left(\omega+\omega_{a}-\omega_{c}\right) \\
& +\frac{4 \pi^{2} \Omega^{2} f(0) g(0)\left(\tilde{G}(0)+\tilde{G}_{c}(0)\right)^{2}}{\left\{\Omega^{2}[f(0)+g(0)]+2 f(0) g(0) h(0)\right\}^{2}} \delta(\omega)
\end{aligned}
$$

Depends on the sign of $\omega_{c}-\omega_{L}$, Eq.(42) has following steady state solutions, 


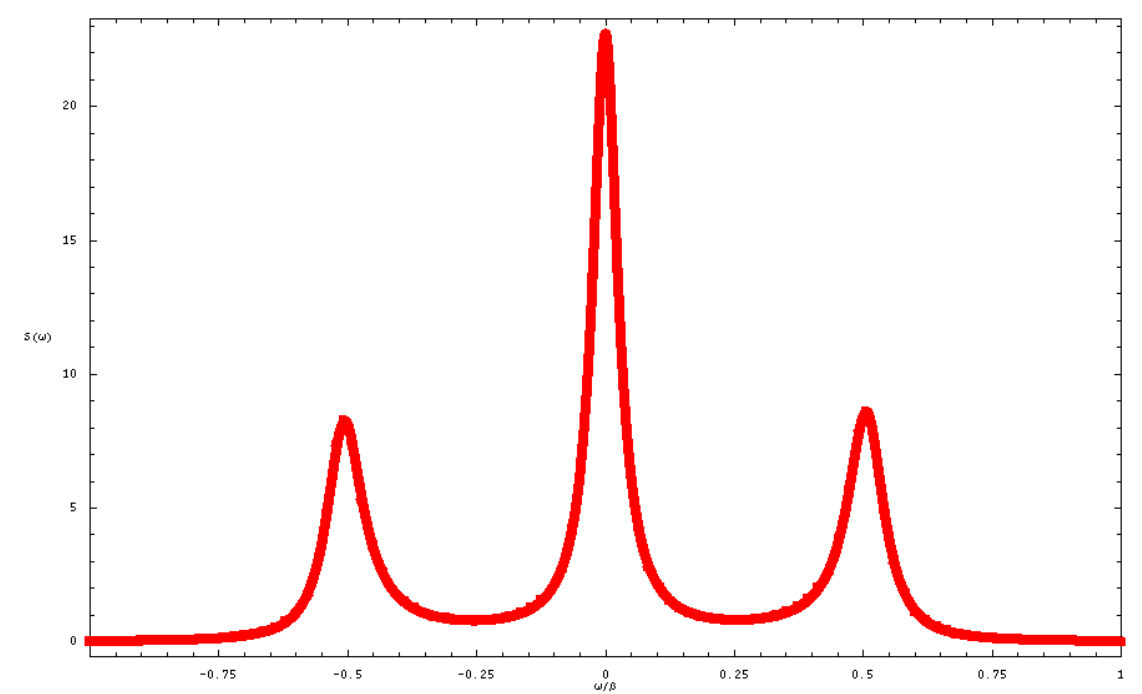

Figure 1. Fluorescence spectrum with resonant pumping $\left(\omega_{a}=\omega_{L}\right)$ when $\omega_{a} \gg \omega_{c} ; \omega_{a}=110 \beta$, and $\omega_{c}=100 \beta$, $\Omega=0.5 \beta$.

for $\omega_{c}-\omega_{L} \geq 0$ :

$$
\begin{aligned}
& \left\langle\tilde{\sigma}_{-}(0)\right\rangle_{R}=0 \\
& \left\langle\tilde{\sigma}_{+}(0)\right\rangle_{R}=0 \\
& \left\langle\tilde{\sigma}_{z}(0)\right\rangle_{R}=0
\end{aligned}
$$

and for $\omega_{c}-\omega_{L}<0$ :

$$
\begin{aligned}
\left\langle\tilde{\sigma}_{-}(0)\right\rangle_{R} & =\frac{i 2 \pi \Omega g(0)\left[\tilde{G}(0)+\tilde{G}_{c}(0)\right]}{\Omega^{2}[f(0)+g(0)]+2 f(0) g(0) h(0)} \\
\left\langle\tilde{\sigma}_{+}(0)\right\rangle_{R} & =\frac{-i 2 \pi \Omega f(0)\left[\tilde{G}(0)+\tilde{G}_{c}(0)\right]}{\Omega^{2}[f(0)+g(0)]+2 f(0) g(0) h(0)} \\
\left\langle\tilde{\sigma}_{z}(0)\right\rangle_{R} & =\frac{2 \pi f(0) g(0)\left[\tilde{G}(0)+\tilde{G}_{c}(0)\right]}{\Omega^{2}[f(0)+g(0)]+2 f(0) g(0) h(0)}
\end{aligned}
$$

In Fig.(1), we plot the resonance fluorescence spectrum at a constant Rabi frequency when the atomic transition frequency is above the bang edge. Here the line-width of each peak is proportional to $\beta^{3 / 2}$. When the atomic transition frequency is far away from the band edge $\left(\omega_{a} \gg \omega_{c}\right)$, the normal resonance fluorescence spectrum of Mollow triplets is obtained just as expected. As the atomic transition frequency moving toward the band edge, asymmetrical Mollow triplets spectra appear. The peak in the lower frequency is suppressed due to the bandgap as shown in Fig.(2). Its residual profile can be with a sharp edge as shown in the inset of the figure. It should also be noted that the peak in the higher frequency is enhanced a lot as can be clearly seen in the Figure. Eventually the peak in the lower frequency will be totally suppressed when the atomic transition frequency is moving more toward the band edge. At this time the resonance fluorescence spectrum now only has two peaks. This is of course due to the bandgap effect. It is interesting to see that now the enhancement of the original middle frequency peak becomes larger then the original higher frequency peak. If the atomic transition frequency is further below the band edge, then eventually only one peak is observed and its profile will also depend on the wavelength offset as shown in Fig.(4(a)-(d)). 


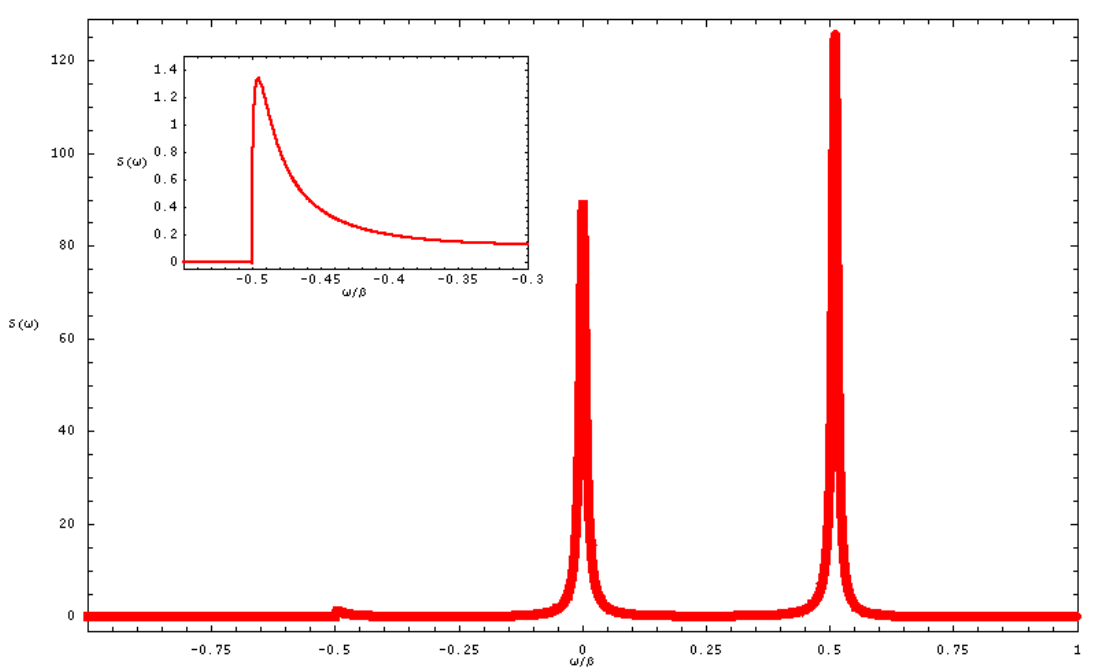

Figure 2. Fluorescence spectrum with $\omega_{a}$ near the band edge; $\omega_{a}=100.5 \beta, \omega_{c}=100 \beta$, and $\Omega=0.5 \beta$; inset: enlarge picture of low-frequency peak.

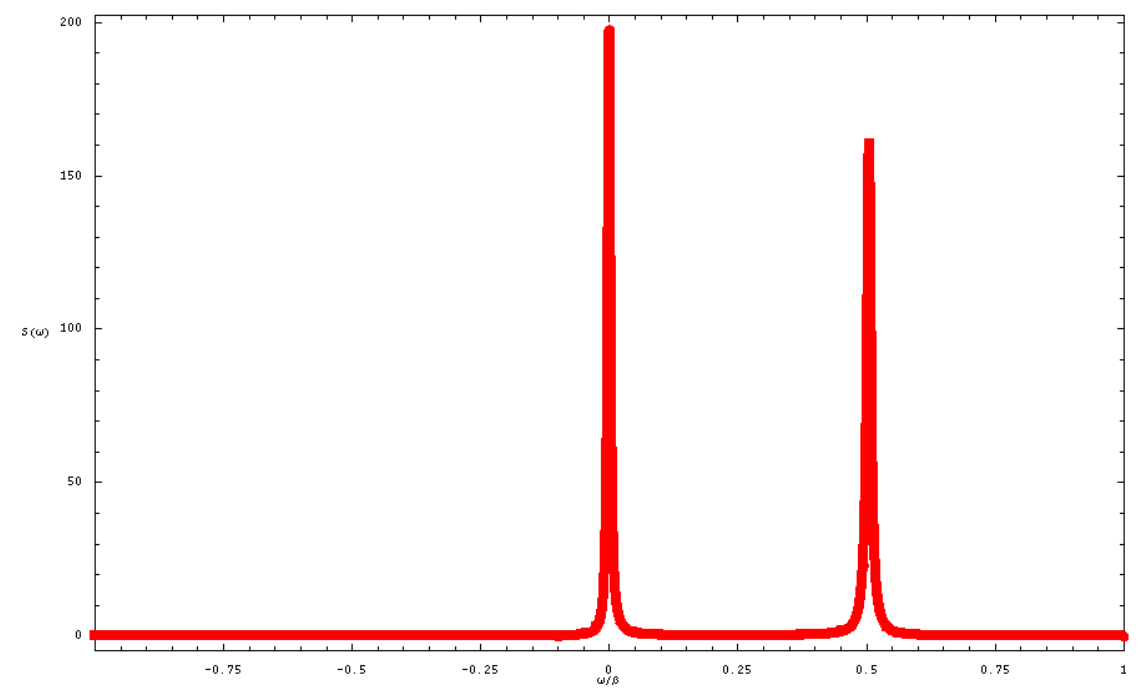

Figure 3. Fluorescence spectrum with $\omega_{a}$ very close to $\omega_{c} ; \omega_{a}=100.1 \beta, \omega_{c}=100 \beta$, and $\Omega=0.5 \beta$. 

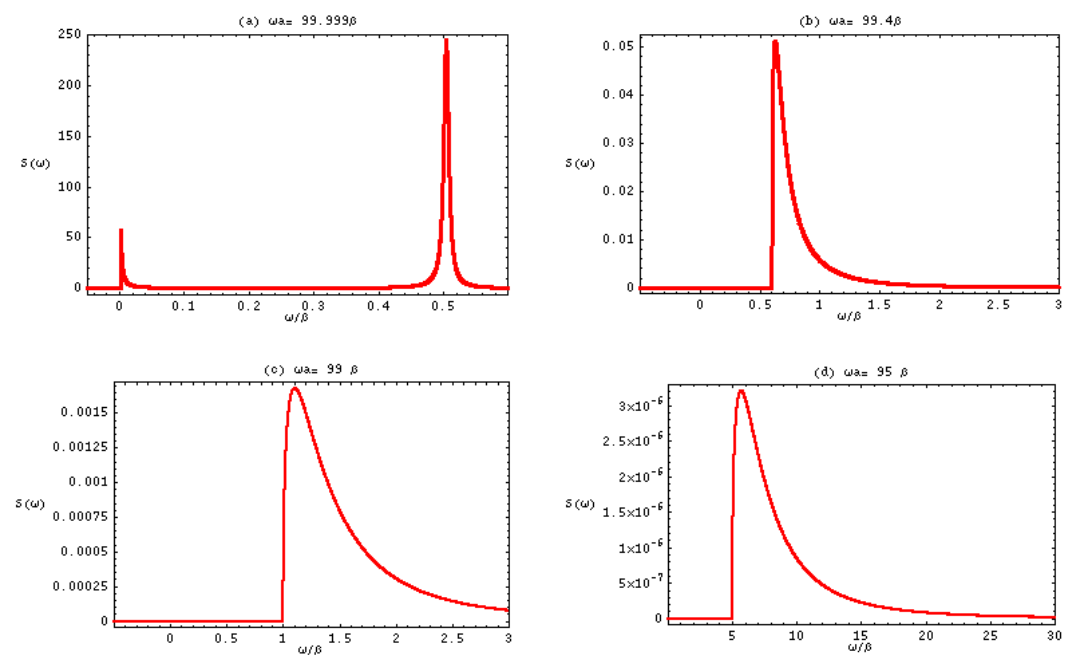

Figure 4. Fluorescence spectrum with $\omega_{a}<\omega_{c} ;(\mathrm{a}): \omega_{a}=99.999 \beta ;(\mathrm{b}): \omega_{a}=99.4 \beta ;$ (c): $\omega_{a}=99 \beta ;(d): \omega_{a}=95 \beta$, and $\omega_{c}=100 \beta, \Omega=0.5 \beta$

\section{CONCLUSIONS}

In conclusion, we have developed a new formulation to calculate the resonance fluorescence spectrum when a single atom is embedded in a photonic bandgap crystal with its transmission wavelength near the photonic band edge. The new approach can overcome the difficulty caused by the non-Markovian nature of the problem due to the non-uniform distribution of the photon states in the photonic bandgap crystal. This has been accomplished by employing the zero-th order Liouville operator expansion to simplify the nonlinear Bloch equations into linear ones and directly calculate the correlation functions of the atomic operators in the Fourier domain. The calculated results have indicated that the resonance fluorescence spectrum near a photonic bandgap can exhibit interesting behavior including the suppression and enhancement of the relative fluorescence peak amplitudes at different wavelength offsets. It shall be very interesting to see if one can actually observe these new phenomena experimentally.

\section{ACKNOWLEDGMENTS}

One of the authors (R. L.) is grateful to Dr. Zheng Yao Su and Dr. Yu-Li Lee for a number of useful suggestions.

\section{REFERENCES}

1. E. M. Purcell, "Spontaneous Emission Probabilities at Radio Frequencies," Phys. Rev. 69, pp. 681, 1946.

2. S. John, "Strong localization of photons in certain disordered dielectric superlattices," Phys. Rev. Lett. 58, pp. $2486,1987$.

3. E. Yablonovitch, "Inhibited Spontaneous Emission in Solid-State Physics and Electronics," Phys. Rev. Lett. 58, pp. 2059, 1987.

4. S. John and T. Quang, "Quantum electrodynamics near a photonic band gap: Photon bound states and dressed atoms," Phys. Rev. Lett. 64, pp. 2418, 1990.

5. S. John and T. Quang, "Spontaneous emission near the edge of a photonic band gap," Phys. Rev. A 50, pp. 1764, 1994.

6. Shi-Yao Zhu, Hong Chen, and Hu Huang, "Quantum Interference Effects in Spontaneous Emission from an Atom Embedded in a Photonic Band Gap Structure," Phys. Rev. Lett. 79, pp. 205, 1997. 
7. E. Paspalakis and P. L. Knight, "Phase Control of Spontaneous Emission," Phys. Rev. Lett. 81, pp. 293, 1998.

8. E. Paspalakis and P. L. Knight, "Transparency near a photonic band edge," Phys. Rev. A 60, pp. R33, 1999.

9. M. Florescu and S. John, "Single-atom switching in photonic crystals," Phys. Rev. A 64, pp. $033801,2001$.

10. R. R. Mollow, "Power Spectrum of Light Scattered by Two-Level Systems," Phys. Rev. 188, pp. $1969,1969$.

11. Shi-Yao Zhu, Yaping Yang, Hong Chen, Hang Zheng, and M. S. Zubairy, "Spontaneous Radiation and Lamb Shift in Three-Dimensional Photonic Crystals," Phys. Rev. Lett. 84, pp. 2136, 2000.

12. Ray-Kuang Lee and Yinchieh Lai, to be published. 